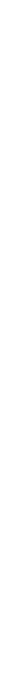

My memories - Meine Erinnerungen an Günter Hager 1986 - 2017

$\begin{aligned} \text { Authors: } & \text { Erich Schanze } \\ \text { Submitted: } & \text { 20. March } 2018 \\ \text { Published: } & \text { 1. April } 2018 \\ \text { Volume: } & 5 \\ \text { Issue: } & 3 \\ \text { Languages: } & \text { German } \\ \text { Keywords: } & \text { memories, literatur, law } \\ \text { DOl: } & 10.17160 / \text { josha.5.3.401 }\end{aligned}$

Journal of Science, Humanities and Arts

JOSHA is a service that helps scholars, researchers, and students discover, use, and build upon a wide range of content 


\title{
Meine Erinnerungen an Günter Hager 1986 - 2017
}

\author{
von Erich Schanze
}

Mit der Erfahrung des Todes eines Freundes sind glücklichere Menschen erst im späteren Lebensalter konfrontiert. Aber auch hier: Die Nachricht macht sprachlos. Floskeln und Formeln ersetzen die Sprache. Sprachbewusste drängt es zur Flucht. „Trauerarbeit“ ist nur eine Antwort der instrumentellen Vernunft. Erst später treten aus dem Nebel der Erinnerung Gestalten und deren Örter hervor, topoi. Die Erörterung der Erinnerung wird im Abstand wieder sprachlich gestaltbar. So erscheint in dem folgenden Bericht Günter Hager in der Topografie meiner deutschen Wanderjahre der letzten 30 Jahre.

\section{Marburg 1986/7}

Günter Hager kannte ich aus der Fachliteratur zum Kaufrecht. Persönlich habe ich ihn anlässlich meiner Lehrstuhlvertretung (Lehrstuhl Wolf, Arbeitsrecht) in Marburg im Wintersemester 1986/7 kennengelernt. Er hatte mich zur Vertretung angeheuert. Am 2. Juni 1986 finde ich einen Kalendereintrag „Hager anrufen“. Überwiegend war ich am Dienstag und Mittwoch in Marburg mit 7 Vorlesungsstunden, davon 3 Stunden Kollektives Arbeitsrecht, 2 Stunden Wirtschaftsrecht und 2 Std. BGB für Wirtschaftswissenschaftler mit Übungen plus vielen Abschlussexamen und deren Wiederholung. Damals war ich als Privatdozent noch Anwalt und führte neben der ständigen Beratung eines schwedischen Unternehmens u.a. einen größeren gesellschaftsrechtlichen Prozess mit mehreren Verfahren vor dem Landgericht und Oberlandesgericht Frankfurt. Die Kinder waren 6 und 4. Außerdem war ich auf Konferenzen zur Neuen Politischen Ökonomie und der Institutionenökonomik unterwegs und hatte rege Kontakte mit ehemaligen Kollegen aus den USA.

Wir freundeten uns rasch an und trafen uns regelmäßig am Dienstag zum gemeinsamen Abendessen in den verschiedenen Wirtschaften der Altstadt. Öfter wurde es spät, bevor ich im Gästehaus der Uni im Alten Botanischen Garten ins Bett kam. Er wohnte damals noch allein in der Rudolf-Klapp-Straße mit bester Aussicht auf die Stadt und das Marburger Schloss. Da wir beide überzeugte Rechtsvergleicher waren, hatten wir ähnliche Ansichten und Einschätzungen, nicht nur zum Stand des Faches, sondern auch zu den Kollegen. Köstlich waren die Berichte über den „Alten“, wie er seinen Lehrer und Mentor Ernst von Caemmerer (auch kurz: „vC“) liebevoll nannte, ebenso über die notorischen ehemaligen vC-Assistenten mit ihren Stärken und 
Schwächen. Er konnte sie allesamt, Chef und Adlaten, trefflich und mit größtem Vergnügen imitieren, auch seinen Studienfreund Jürgen Schwarze, mit dem er gemeinsam die vorzüglichen Examen vorbereitet hatte. Mit dem Kopf lebte er wohl noch überwiegend in Freiburg. Dies spiegelte sich auch in der Besetzung der beiden rechtsvergleichenden Lehrstühle: der ältere vCSchüler Hans Leser in der „Anglo-amerikanischen Abteilung“, er selbst, der jüngste „persönliche Assistent“ vCs (wie er es stolz differenzierend erklärte), in der „Allgemeinen Abteilung“. Marburg war so gesehen eine Nebenstelle der Freiburger vC-Schule. Natürlich musste ich aus dem nachrevolutionäreren Frankfurt berichten, und von meinen vielen Aufenthalten in den USA. Er beriet mich bei verschiedenen Vorstellungsgesprächen an anderen deutschen Fakultäten. Er kannte sich da viel besser aus.

Eines der Gesprächsthemen war die Nominierung eines neuen Marburger UniversitätsPräsidenten. Günter Hager war sowohl bei der sog. Professorenliste als auch bei der traditionellen Linken gut vernetzt, und man hatte ihn gefragt, ob er nicht kandidieren wolle. Das passte überhaupt nicht in seinen Lebensplan. Wir diskutierten eine mögliche Präsidentschaft des Romanisten Dietrich Simon, der dann auch prompt gewählt wurde. Hager war sicher ein wichtiger, wenn nicht sogar der entscheidende Faktor bei dieser Wahl.

In der Marburger Fakultät war er ein unabhängiger Geist. Er war aber auch dort ein PowerBroker, denn man schätzte seinen Witz, seinen Arbeitseinsatz und seinen Gerechtigkeitssinn. Er drückte sich nie. Seine Vorlesungen waren „Kult“ im Zivilrecht. Er achtete aber auch auf die „Seniorität“" von Volker Beuthien und Hans Leser, was diese wiederum sehr genossen. Dass er sich angesichts seiner Beliebtheit im Stillen bisweilen vielleicht nicht als „Junior“ verstand, ließ er sich nicht anmerken. Konflikte mochte er nicht. Er gab gern den jugendlichen Helden; wahrscheinlich auch zum Vergnügen der wehmutsvoll zurückblickenden „Senioren“.

\section{Heidelberg 1987-1989}

In dieser ersten Marburger Zeit wurde ich nach Heidelberg berufen, wo ich im Sommersemester 1987 begann. Ab März wohnte ich in der Vorlesungszeit im Hölderlinweg 2. Am 5. März der Antrittsbesuch im Hause Ulmer in der Albert-Überle-Straße. Vorlesungsbeginn am 21. April. Dienstzimmer im Erdgeschoß des ehemaligen Hauses Samuel Pufendorffs (man sagte mir: sein Wohnzimmer) neben der Alten Universität. Im Juni habe ich dort Günter Hager getroffen. Wir haben immer wieder telefoniert.

Im September dann die gemeinsame Reise zu den Zivilrechtslehrern nach Innsbruck. Er war am Vorabend mit dem Zug zu uns nach Schwalbach am Taunus gekommen. Wir hatten noch 
einen besseren Rheingauer Riesling probiert, obwohl er im Allgemeinen „Rote“ bevorzugte. Bei der sonnigen Autofahrt - wir nahmen den Weg durchs Allgäu über den Fernpass - haben wir bereits viel „geratscht“ (einer seiner Lieblingsausdrücke) und gelacht. Als es uns bei den Vorträgen zu langweilig wurde, sind wir Richtung Italien aufgebrochen und einen Tag auf der Seiser Alm in der Sonne herumgewandert.

Damals haben wir auch intensiver über einen möglichen Aufenthalt im Forschungssemester in Chicago als Max-Rheinstein-Gastprofessor gesprochen. Es war sein erster US-Aufenthalt. Er war später noch einmal in Texas. Aber so richtig wohl hat er sich dort nie gefühlt. Er fühlte sich vom Lebensstil nicht angezogen und hatte auch immer einige Bedenken, da seine sprachliche Stärke eindeutig bei Französisch lag. Als „Mathematiker“ war mein klappriges einjähriges Schulfranzösisch allenfalls durch das Skifahren in Les Deux Alpes und Atlantikaufenthalte gerade so gangbar, eher schwach. Natürlich konsultierte ich ihn, als ich im März 1988 in Montpellier einen französischen Vortrag halten musste, dessen Fragen ich dann allerdings vorsichtshalber auf Englisch beantwortete.

Am 8. März 1989 besuchte mich Günter noch einmal in Heidelberg. Da stand allerdings schon fest, dass ich vom Sommersemester an in Oldenburg in dem neuen Mischstudiengang „Betriebswirtschaft mit juristischen Schwerpunkt“ unterrichten würde.

\section{Oldenburg 1989-1996}

Allein durch die Distanz kam es zu selteneren persönlichen Begegnungen. Allerdings tauschten wir Arbeitspapiere aus und trafen uns regelmäßig bei Tagungen. Günter beschäftigte sich inzwischen heftig mit dem Umwelthaftungsrecht, und wir diskutierten seinen Ruf nach Jena. Der Präsident der Universität Oldenburg schickte mich zur Wiedereröffnung als Stellvertreter an die Schiller-Universität, wo zu meinem Erstaunen auch Corps-Studenten den Einzug der damals im Westen weitgehend verschwundenen Talare begrüßten. Bei der Antrittsvorlesung Hagers am 27. November 1992 wurde vor der Veranstaltung noch einmal das Thema „korrekte Behandlung des Baretts bei Begrüßung und Vorlesung“ durchgenommen, wobei ich zum Amüsement meines Freundes auf „wenn schon, denn schon“ insistierte. Er natürlich: „Gerade Du!“

Legendär wurden Günter Hagers Jenenser Seminare mit feuchtem Ausklang. Seine Berichte wurden später immer neu ausgeschmückt und gestisch veredelt. Der absolute Höhepunkt (später wurde das buchstabierte Ende ein running gag) muss die Beschaffung von Trinkbarem zu später Stunde gewesen sein. Als man mitternächtlich trockenfiel - so der Bericht - wurde 
ein Taxi bestellt. Fahrtziel war die einzig offene Autobahntankstelle Jenas (und zurück), natürlich ohne Fahrgast, aber zurück mit Stoff. Der verdutzte Taxi-Fahrer wurde zur der Durchführung des ungewöhnlichen, auch an Kilometern nicht unerheblichen VorkasseAuftrags von Professor Hager selbst klar und deutlich über den Gegenstand der Beschaffung instruiert: „Drei Flaschen Chablis - ich wiederhole: Chablis - ich buchstabiere (bereits bei diesem Wort hob er in unnachahmlicher Weise die Stimme an): C-H-A-B-L-I-S““.

Da ich von Karl Meessen zur externen Mitarbeit an dessen Forschungsschwerpunkt - und damit verbunden - einzelnen Vorlesungsblöcken zu „law and economics“ gebeten worden war, kam ich häufiger nach Jena, insbesondere in der Zeit, als ich schon 1996 nach Marburg gewechselt war. Besonders liebte Günter in Jena den kleinen Stadtturm in der Nähe des Zeiss-Turms. Der habe so eine niedliche, freundliche Kapuze. Es ist ihm in Wahrheit gar nicht leichtgefallen, von Jena nach Freiburg zu wechseln. Denn Jena war für ihn die große Freiheit und ein Stück Exotik. Er wusste: In Freiburg bestand die Gefahr, irgendwie wieder in die Hierarchie des „Alten“ eingereiht zu werden.

\section{Marburg 1996-2017}

Günter Hager kam nicht nur zu meiner Antrittsvorlesung nach Marburg. Seine freundschaftliche Geste, die er gelegentlich wiederholte: „Es ist in Marburg eigentlich doch am schönsten gewesen". Er begründete dies auch. Und irgendwie wirkte es glaubhaft. Köln war eine Episode, Jena ein Abenteuer. In Marburg hatte er Bettina geheiratet. Aufs 700 Jahre alte Schloss geschaut. Sein Sohn wurde dort geboren, den er nach dem Gründer der Universität Philipp nannte. Die Fakultät war eine der streitfreisten der Republik. Man konnte dort gut arbeiten. Es gab immer eine Handvoll guter Studenten. Aber es war ebenso klar: Freiburg war das Ziel. Mit Ausflügen nach Straßburg, mit gelegentlichen Ritten über den Bodensee, im Dunstkreis des „Alten“, am Nabel der Privatrechtsvergleichung.

Seine späteren Besuche hatten andere Gründe. Natürlich war er bei der Savigny-Vorlesung dabei. Richard Posner sprach in der Alten Aula über den Besitz bei Savigny und Holmes. Hagers damaliger Freiburger Assistent Florian Bentele spielte Beethoven c-Moll opus 13 genau 200 Jahre nach der Erstaufführung dieses ungewöhnlichen Künstlerstücks. Festlicher Abend im Rittersaal des Landgrafenschlosses. Das gefiel ihm.

Auch wenn beispielsweise David Currie, Kenneth Dam oder John Langbein in Marburg waren, versuchte er zu kommen. Der Hauptgrund der Marburger Besuche war schwieriger und mir zunächst auch nicht bekannt. Wegen seiner chronischen Krankheit kam GH mehrmals im Jahr 
in die Universitäts-Klinik, bis er bei einem Freund in Freiburg eine besondere Betreuung fand. Auch deshalb sahen wir uns häufiger auf der Kirchspitze und machten Waldspaziergänge.

Die Universität, wie er spöttisch bemerkte, sei ein Zentrum des Menschenhandels. Er kannte den Markt genau und verfolgte die Karrieren. Wenn es um Listengutachten ging, brauchte man nicht lange mit ihm zu handeln. Die Gutachten kamen in Kürze, professionell und mit höchster Sachkunde. Er hatte einen scharfen Blick für Talente bei sehr hohen Maßstäben.

Wie immer war er mit Neuem beschäftigt. Er wollte schon immer Klavierspielen können - und lernte es diszipliniert. Er bedauerte, nicht Altgriechisch zu beherrschen, heuerte einen Lehrer an, und studierte die Sprache in vielstündigen Übungen. Er war von den Lautungen, der Variationsbreite und Ausdruckskraft dieser Sprache begeistert. Später besuchte er als Protestant häufig Gottesdienste beider Konfessionen, nahm an theologischen Vorlesungen teil und las mit größtem Interesse das Jesusbuch des emeritierten Papstes.

Über seine eigenen Buchprojekte haben wir uns intensiv unterhalten. Sie waren für ihn lebenswichtig. Meine Kinder wussten schon, wenn Günter anrief, oder ich bei ihm, dann dauerte es etwas länger. Es wurde ,geratscht“. Nach dem Nachrichtenaustausch über Politik und Familie, möglicherweise auch über die Marburger Riesling-Ernte, beschimpften wir uns gegenseitig mäßig und schlossen aber schnell wieder Frieden. In Freiburg wurde sein Sohn Philipp in der Wohngemeinschaft eine Art jüngerer Bruder. Unvergesslich, wie sich Günter am Telefon meldete, mit einem inzwischen wieder leicht badisch klingenden Singsang (er kam ja eigentlich in der Jugend aus Franken) und einem Lächeln, welches man durch die Leitung hören konnte: „Hier ist der Günter“.

Felix Maultzsch, der auch von Dieter Simon betreute „Hochbegabte“ aus Jena, kam zu meiner Freude zeitweilig ans Marburger Institut, bevor ihm Hager eine Habilitationsstelle in Freiburg anbot. Das Hauptwerk „Rechtsmethoden in Europa“ von 2009 habe ich in der führenden einschlägigen Zeitschrift eingehend kritisch rezensiert. Es war eine Art Einlösung eines scherzhaften wechselseitigen Versprechens, nämlich dass wir uns in der Akme unserer späten Tage eigentlich nur noch mit ein bis zwei giftigen jährlichen Rezensionen in altertümlichen Zeitschriften wie dem „Archiv für die civilistische Praxis“ oder „Rabels Zeitschrift“ die Zeit vertreiben sollten. Aber: was wären die Bücher, die unsere Aufmerksamkeit verdienten, schrieben wir sie nicht selbst?

Günter Hager hat noch zwei weitere Bücher geschrieben - und war insoweit vielleicht näher an unserem Teufelspakt. Ganz wichtig war ihm das letzte Büchlein zum Recht der Tiere. Die 
Behandlung der Tiere, die unserer Lebensweise eigen ist, ist Unrecht. Rechtfertigungen sind kaum möglich. Die Indifferenz macht betroffen. Wir wissen, dass Nietzsche am Lebensende ähnlich dachte und haben dies, um uns zu schützen, fragwürdig mit Schwäche erklärt.

Vielleicht noch wichtiger war ihm der juristische Kommentar zur Enzyklika „laudato sí “ von Papst Franziskus in der Internet-Zeitschrift JOSHA. Er sieht in der Enzyklika seinen früher geäußerten Gedanken bestätigt, dass der Mensch gegenüber der Natur das Maß verloren hat. Eine Wende sei das Gebot der Stunde. Auch Juristen sollten dies beherzigen.

Bisweilen greife ich noch zum Telefon und möchte Günter Hager anrufen. Er war ein seltener Freund. Im letzten Kapitel der „Rechtsmethoden in Europa“ lenkt er den Blick von den „Rechtsmethoden“ zu dem Menschen, der mit ihnen befasst ist. Er entwickelt dabei den Topos vom ,guten Juristen“. Manche mögen sagen, dies sei eine contradictio in adiecto. Aber: Günter Hager war nun einmal unbestreitbar ein guter Jurist.

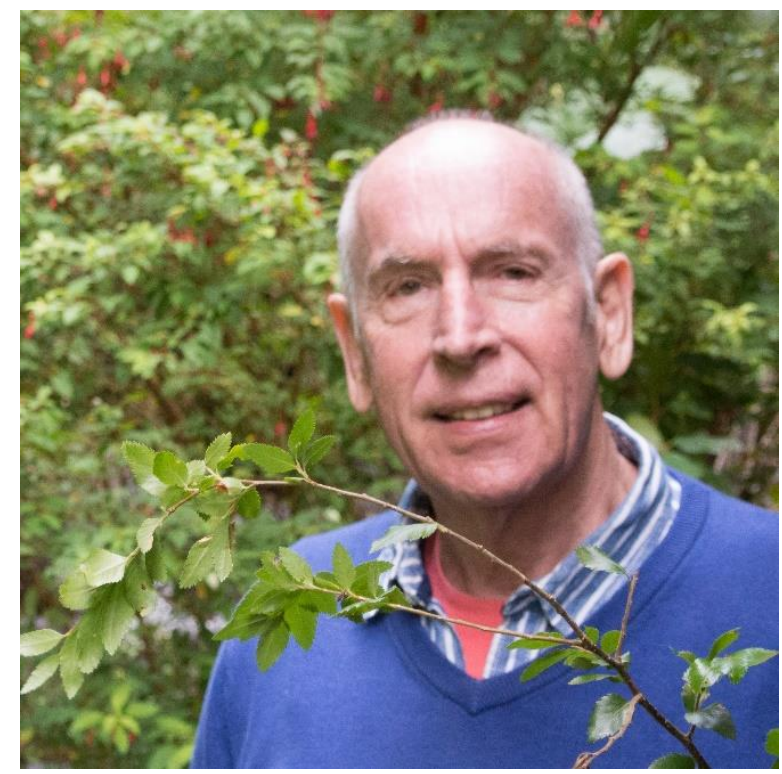

Professor Dr. Dr. h.c. Erich Schanze LL.M.
Geboren 1942, Studium in Frankfurt, Edinburgh und Harvard (dort LL.M. 1969).

Ist seit 1995 Professor für Bürgerliches Recht, Rechtsvergleichung und Internationales Wirtschaftsrecht in Marburg. Direktor des Instituts für Rechtsvergleichung - AngloAmerikanische Abteilung; seit 2004 Lehrauftrag für Law and Economics, Universität St. Gallen; seit 2006 Professor II (ständige Gastprofessur), Det juridiske fakultet, Universitetet i Bergen.

\section{(Harv.)}

